J. Dairy Sci. 102:6699-6699

https://doi.org/10.3168/jds.2019-7-6699

(c) American Dairy Science Association ${ }^{\circledR}, 2019$.

\title{
Corrigendum to "Changes in fermentation and animal performance during recovery from classical diet-induced milk fat depression using corn with differing rates of starch degradability" (J. Dairy Sci. 102:5079-5093)
}

\section{E. Koch, T. C. Jenkins, W. C. Bridges, B. M. Koch, and G. J. Lascano}

An error was noted in the reported values for total protozoa counts in Table 6 (page 5091). The corrected table (corrected values in bold) is shown below. A treatment effect followed the same pattern as reported previously but at $P=0.04$ instead of $P<0.01$.

The authors regret the error.

Table 6. Protozoal populations of ruminal contents during recovery from diet-induced milk fat depression using a low degradable starch (LDS) or high degradable starch (HDS) diet

\begin{tabular}{|c|c|c|c|c|c|c|c|c|}
\hline Protozoa, ${ }^{1} 10^{4} / \mathrm{mL}$ & $\begin{array}{l}\text { Treatment } \\
\text { (Trt) }\end{array}$ & \multicolumn{3}{|c|}{ Day during recovery } & SEM & \multicolumn{3}{|c|}{$P$-value } \\
\hline \multirow[t]{2}{*}{ Total } & HDS & $82.8^{*}$ & $81.7^{*}$ & $75.2^{*}$ & 1.52 & $<0.01$ & 0.04 & $<0.01$ \\
\hline & LDS & 71.6 & 73.6 & 70.0 & & & & \\
\hline \multirow{2}{*}{ Entodinium spp. } & HDS & $77.3^{*}$ & $76.4^{*}$ & $69.8^{*}$ & 1.37 & $<0.01$ & 0.02 & 0.04 \\
\hline & LDS & 66.8 & 68.7 & 65.4 & & & & \\
\hline Epidinium spp. & HDS & 1.02 & 1.01 & 0.81 & 0.23 & $<0.01$ & 0.13 & 0.37 \\
\hline Diplodinium spp. & LDS & 1.24 & 1.24 & 1.15 & & & & \\
\hline \multirow{2}{*}{ Dasytricha spp. } & HDS & 1.16 & 1.20 & 1.21 & 0.69 & 0.11 & 0.27 & 0.63 \\
\hline & LDS & 1.15 & 1.25 & 1.22 & & & & \\
\hline \multirow[t]{2}{*}{ Isotricha spp. } & HDS & $0.97^{*}$ & 0.79 & 1.17 & 0.77 & 0.45 & 0.89 & 0.63 \\
\hline & LDS & 0.80 & 0.79 & 0.85 & & & & \\
\hline \multirow{2}{*}{ Orphryoscolex spp. } & HDS & 0.64 & 0.59 & 0.75 & 0.10 & 0.17 & 0.30 & 0.31 \\
\hline & LDS & 0.75 & 0.69 & 0.71 & & & & \\
\hline
\end{tabular}

${ }^{1}$ Estimated according to the methods of Dehority (1993).

*Significantly different $(P \leq 0.05)$ at the given time point.

\section{REFERENCES}

Koch, L. E., T. C. Jenkins, W. C. Bridges, B. M. Koch, and G. J. Lascano. 2019. Changes in fermentation and animal performance during recovery from classical diet-induced milk fat depression using corn with differing rates of starch degradability. J. Dairy Sci. 102(6):5079-5093. https://doi.org/10.3168/jds.2018-15953. 